\title{
Some Inverse Relations Determined by Catalan Matrices
}

\author{
Sheng-liang Yang \\ Department of Applied Mathematics, Lanzhou University of Technology, Lanzhou, Gansu 730050, China \\ Correspondence should be addressed to Sheng-liang Yang; slyang@lut.cn
}

Received 24 June 2013; Revised 21 August 2013; Accepted 21 August 2013

Academic Editor: Laszlo A. Szekely

Copyright (C) 2013 Sheng-liang Yang. This is an open access article distributed under the Creative Commons Attribution License, which permits unrestricted use, distribution, and reproduction in any medium, provided the original work is properly cited.

We use the $A$-sequence and $Z$-sequence of Riordan array to characterize the inverse relation associated with the Riordan array. We apply this result to prove some combinatorial identities involving Catalan matrices and binomial coefficients. Some matrix identities obtained by Shapiro and Radoux are all special cases of our identity. In addition, a unified form of Catalan matrices is introduced.

\section{Introduction}

The Catalan numbers $C_{n}$ have been widely encountered and investigated $[1,2]$. They can be defined through binomial coefficients

$$
C_{n}=\frac{1}{n+1}\left(\begin{array}{c}
2 n \\
n
\end{array}\right), \quad \text { for } n \geq 0,
$$

or by the generating function $C(t)=\sum_{n=0}^{\infty} C_{n} t^{n}$ being

$$
C(t)=\frac{1-\sqrt{1-4 t}}{2 t},
$$

which satisfies the functional equation $C(t)=1+t C(t)^{2}$. In [2], Stanley listed 66 enumerative problems which are counted by the Catalan numbers. Many number triangles related to the Catalan sequence have been introduced in the literature. In [3-5], Shapiro et al. introduced a Catalan triangle $B$ with the entries given by

$$
B_{n, k}=\frac{k+1}{n+1}\left(\begin{array}{c}
2 n+2 \\
n-k
\end{array}\right), \quad \text { where } n \geq k \geq 0 .
$$

The following identity is obtained in [6] in connection with the moment of the Catalan triangle:

$$
\left(\begin{array}{cccccc}
1 & 0 & 0 & 0 & 0 & \cdots \\
2 & 1 & 0 & 0 & 0 & \cdots \\
5 & 4 & 1 & 0 & 0 & \cdots \\
14 & 14 & 6 & 1 & 0 & \cdots \\
42 & 48 & 27 & 8 & 1 & \cdots \\
\vdots & \vdots & \vdots & \vdots & \vdots & \ddots
\end{array}\right)\left(\begin{array}{c}
1 \\
2 \\
3 \\
4 \\
5 \\
\vdots
\end{array}\right)=\left(\begin{array}{c}
1 \\
4 \\
4^{2} \\
4^{3} \\
4^{4} \\
\vdots
\end{array}\right)
$$

Another proof of the above identity is given by Woan et al. [7] while computing the areas of parallelo-polyominoes via generating functions. In [8], a combinatorial interpretation of the matrix identity (4) is also obtained.

In [9], Radoux introduced a triangle of numbers

$$
c_{n, k}=\frac{2 k+1}{n+k+1}\left(\begin{array}{c}
2 n \\
n-k
\end{array}\right), \quad \text { where } n \geq k \geq 0,
$$

and he presents the identity $\sum_{k=0}^{n}(2 k+1) c_{n, k}=2^{2 n}$ with $n \geq$ $k \geq 0$, which is equivalent to following matrix equation:

$$
\left(\begin{array}{cccccc}
1 & 0 & 0 & 0 & 0 & \cdots \\
1 & 1 & 0 & 0 & 0 & \cdots \\
2 & 3 & 1 & 0 & 0 & \cdots \\
5 & 9 & 5 & 1 & 0 & \cdots \\
14 & 28 & 20 & 7 & 1 & \cdots \\
\vdots & \vdots & \vdots & \vdots & \vdots & \ddots
\end{array}\right)\left(\begin{array}{c}
1 \\
3 \\
5 \\
7 \\
9 \\
\vdots
\end{array}\right)=\left(\begin{array}{c}
1 \\
4 \\
4^{2} \\
4^{3} \\
4^{4} \\
\vdots
\end{array}\right) .
$$

Deng and Yan [10] proved this identity by using the Riordan array method.

Aigner [11] introduced a number triangle with the entries given by

$$
a_{n, k}=\frac{k+1}{n+1}\left(\begin{array}{c}
2 n-k \\
n-k
\end{array}\right), \quad \text { where } n \geq k \geq 0 .
$$

This array is also discussed in [12-14].

We use the $A$-sequence and $Z$-sequence of Riordan array to characterize the inverse relation associated with the 
Riordan array. We apply this result to prove some combinatorial identities involving Catalan matrices and binomial coefficients, which are generalizations of (4) and (6). In addition, a unified form of Catalan matrices is introduced.

\section{Riordan Arrays}

In the recent literature, one may find that Riordan arrays have attracted the attention of various authors from many points of view, and many examples and applications can be found (see, e.g., [13, 15-21]). An infinite lower triangular matrix $D=\left(d_{n, k}\right)_{n, k \geq 0}$ is called a Riordan array if its column $k$ has generating function $g(t) f(t)^{k}$, where $g(t)$ and $f(t)$ are formal power series with $g_{0}=1, f_{0}=0$, and $f_{1} \neq 0$. The Riordan array is denoted by $D=(g(t), f(t))$. Thus, the general term of Riordan array $D=(g(t), f(t))$ is given by

$$
d_{n, k}=\left[t^{n}\right] g(t) f(t)^{k}
$$

where $\left[t^{n}\right] h(t)$ denotes the coefficient of $t^{n}$ in power series $h(t)$. Suppose we multiply the array $D=(g(t), f(t))$ by a column vector $\left(b_{0}, b_{1}, b_{2}, \ldots\right)^{T}$ and get a column vector $\left(a_{0}, a_{1}, a_{2}, \ldots\right)^{T}$. Let $b(t)$ be the ordinary generating function for the sequence $\left(b_{0}, b_{1}, b_{2}, \ldots\right)^{T}$. Then it follows that the ordinary generating function for the sequence $\left(a_{0}, a_{1}, a_{2}, \ldots\right)^{T}$ is $g(t) b(f(t))$. If we identify a sequence with its ordinary generating function, the composition rule can be rewritten as

$$
(g(t), f(t)) b(t)=g(t) b(f(t)) .
$$

This is called the fundamental theorem for Riordan arrays, and this leads to the multiplication rule for the Riordan arrays:

$$
(g(t), f(t))(h(t), l(t))=(g(t) h(f(t)), l(f(t))) .
$$

The set of all Riordan arrays forms a group under ordinary multiplication. The identity is $(1, t)$. The inverse of $(g(t), f(t))$ is

$$
(g(t), f(t))^{-1}=\left(\frac{1}{g(\bar{f}(t))}, \bar{f}(t)\right),
$$

where $\bar{f}(t)$ is compositional inverse of $f(t)$.

Lemma 1 (see $[22,23])$. Let $D=\left(d_{n, k}\right)$ be an infinite lower triangular matrix. Then $D$ is a Riordan array if and only if $d_{0,0}=1$ and there exist two sequences $A=\left(a_{i}\right)_{i \geq 0}$ and $Z=\left(z_{i}\right)_{i \geq 0}$ with $a_{0} \neq 0$ and $z_{0} \neq 0$ such that

$$
\begin{aligned}
d_{n+1, k+1}= & a_{0} d_{n, k}+a_{1} d_{n, k+1} \\
& +a_{2} d_{n, k+2}+\ldots, \quad n, k=0,1, \ldots, \\
d_{n+1,0}= & z_{0} d_{n, 0}+z_{1} d_{n, 1}+z_{2} d_{n, 2}+\ldots, \quad n=0,1, \ldots
\end{aligned}
$$

Such sequences are called the A-sequence and the $Z$-sequence of the Riordan array $D=(g(t), f(t))$, respectively.
Lemma 2 (see $[22,23])$. Let $D=(g(t), f(t))$ be a Riordan array, and let $A(t)$ and $Z(t)$ be the generating functions for the corresponding $A$ - and $Z$-sequences, respectively. Then we have

$$
g(t)=\frac{1}{1-t Z(f(t))}, \quad f(t)=t A(f(t)) .
$$

If the inverse of $D=(g(t), f(t))$ is $D^{-1}=(d(t), h(t))$. Then

$$
A(t)=\frac{t}{h(t)}, \quad Z(t)=\frac{1-d(t)}{h(t)} .
$$

Example 3. (a) It is well known that the Pascal matrix $P=$ $\left(\left(\begin{array}{c}i \\ j\end{array}\right)\right)_{i, j \geq 0}$ can be expressed as the Riordan array $(1 /(1-$ $t), t /(1-t))$, and the generating functions of its $A$ - and $Z$-sequences are $A(t)=1+t, Z(t)=1$. More generally, it is easy to show that the generalized Pascal array $P[x]=$ $\left(x^{i-j}\left(\begin{array}{c}i \\ j\end{array}\right)\right)_{i, j \geq 0}$ can be expressed as the Riordan matrix $(1 /(1-$ $x t), t /(1-x t))$ and the generating functions of its $A$ - and $Z$-sequences are $A(t)=1+x t$, and $Z(t)=x$.

(b) For nonnegative integer $s$, the Pascal functional $s$-eliminated matrix $P_{s}$ was introduced in [24] by $P_{s}=$ $\left(x^{i-j}\left(\begin{array}{c}i+s \\ j+s\end{array}\right)\right)_{i, j \geq 0}$. We have $P_{s}=\left(1 /(1-x t)^{s+1}, t /(1-x t)\right)$, and the generating functions of its $A$ - and $Z$-sequences are $A(t)=1+x t, Z(t)=\left((1+x t)^{s+1}-1\right) /\left(t(1+x t)^{s}\right)$. We also have $P_{s}^{-1}=\left(1 /(1-x t)^{s+1}, t /(1-x t)\right)^{-1}=\left(1 /(1+x t)^{s+1}, t /(1+x t)\right)$.

Definition 4. Let $\left(r_{n}(x)\right)_{n \geq 0}$ be a sequence of polynomials, where $r_{n}(x)$ is of degree $n$ and $r_{n}(x)=\sum_{k=0}^{n} r_{n, k} x^{k}$. We say that $\left(r_{n}(x)\right)_{n \geq 0}$ is a polynomial sequence of Riordan type if the coefficient matrix $\left(r_{n, k}\right)_{n, k \geq 0}$ is an element of the Riordan group; that is, there exists a Riordan array $(g(t), f(t))$ such that $\left(r_{n, k}\right)_{n, k \geq 0}=(g(t), f(t))$. In this case, we say that $\left(r_{n}(x)\right)_{n \geq 0}$ is the polynomial sequence associated to the Riordan array $(g(t), f(t))$.

If $\left(r_{n}(x)\right)_{n \geq 0}$ is the polynomial sequence associated to a Riordan array $(g(t), f(t))$, and let $r(t, x)=\sum_{n=0}^{\infty} r_{n}(x) t^{n}$ be its generating function, then by (9), we have

$$
(g(t), f(t)) \frac{1}{1-x t}=r(t, x) \text {. }
$$

Thus, $r(t, x)=g(t) /(1-x f(t))$. The notion of the polynomial sequence of Riordan type was introduced in [25], and it has been studied by [26]. In this paper, by studying the polynomial sequence of Riordan type related to some Catalan type matrices, we obtain some interesting identities and inverse relations.

Theorem 5. Let $D=(g(t), f(t))$ be a Riordan array, and let $A(t)$ and $Z(t)$ be the generating functions of its $A$-sequence and $Z$-sequence. If $B(t)=(A(t)-t Z(t)) /(A(t)-x t)$, then

$$
(g(t), f(t)) B(t)=\frac{1}{1-x t}
$$

where $x$ is any real number. 
Proof. Let $B(t)=(A(t)-t Z(t)) /(A(t)-x t)$; then $(g(t)$, $f(t)) B(t)=g(t) B(f(t))=(1 /(1-t Z(f(t))))((A(f(t))-$ $f(t) Z(f(t))) /(A(f(t))-x f(t)))=(1 /(1-t Z(f(t))))((f(t)-$ $t f(t) Z(f(t))) /(f(t)-x f(t)))=1 /(1-x t)$.

Corollary 6. Let $D=(g(t), f(t))$ be a Riordan array. If $A(t)$ and $Z(t)$ are the generating functions of its $A$ sequence and $Z$-sequence, respectively, and if $\left(\varphi_{n}(x)\right)_{n \geq 0}$ is the polynomial sequence associated to the Riordan array $D^{-1}=$ $(1 / g(\bar{f}(t)), \bar{f}(t))$, then

$$
\sum_{n=0}^{\infty} \varphi_{n}(x) t^{n}=\frac{A(t)-t Z(t)}{A(t)-x t} .
$$

Proof. By the theorem, we have

$$
(g(t), f(t)) \frac{A(t)-t Z(t)}{A(t)-x t}=\frac{1}{1-x t} .
$$

Hence,

$$
(g(t), f(t))^{-1} \frac{1}{1-x t}=\frac{A(t)-t Z(t)}{A(t)-x t} .
$$

The result then follows from identity (15).

Corollary 7. Let $D=\left(d_{n, k}\right)$ be a Riordan array. If $A(t)$ and $Z(t)$ are the generating functions of its $A$-sequence and $Z$-sequence, respectively, and if $\left(\varphi_{n}(x)\right)_{n \geq 0}$ is the polynomial sequence associated to the Riordan array $D^{-1}$, then

$$
\begin{aligned}
& \sum_{k=0}^{n} d_{n, k} \varphi_{k}(x)=x^{n}, \\
& \sum_{k=0}^{n} \bar{d}_{n, k} x^{k}=\varphi_{n}(x),
\end{aligned}
$$

where $\bar{d}_{n, k}$ is the $(n, k)$-element of $D^{-1}$. In matrix form, we have

$$
\begin{gathered}
\left(\begin{array}{cccccc}
d_{0,0} & 0 & 0 & 0 & 0 & \cdots \\
d_{1,0} & d_{1,1} & 0 & 0 & 0 & \cdots \\
d_{2,0} & d_{2,1} & d_{2,2} & 0 & 0 & \cdots \\
d_{3,0} & d_{3,1} & d_{3,2} & d_{3,3} & 0 & \cdots \\
d_{4,0} & d_{4,1} & d_{4,2} & d_{4,3} & d_{4,4} & \cdots \\
\vdots & \vdots & \vdots & \vdots & \vdots & \ddots
\end{array}\right)\left(\begin{array}{c}
\varphi_{0}(x) \\
\varphi_{1}(x) \\
\varphi_{2}(x) \\
\varphi_{3}(x) \\
\varphi_{4}(x) \\
\vdots
\end{array}\right) \\
=\left(\begin{array}{c}
1 \\
x \\
x^{2} \\
x^{3} \\
x^{4} \\
\vdots
\end{array}\right) .
\end{gathered}
$$

Example 8. The lower triangular matrix in (4) may be represented as

$$
\left(C(t)^{2}, t C(t)^{2}\right)=\left(\frac{1-2 t-\sqrt{1-4 t}}{2 t^{2}}, \frac{1-2 t-\sqrt{1-4 t}}{2 t}\right) .
$$

The generating functions of its $A$ - and $Z$-sequence are $A(t)=$ $1+2 t+t^{2}, Z(t)=2+t$. Because $(A(t)-t Z(t)) /(A(t)-4 t)=$ $1 /(1-t)^{2}=\sum_{n=0}^{\infty}(n+1) t^{n}$, from Theorem 5 , we have

$$
\left(C(t)^{2}, t C(t)^{2}\right) \frac{1}{(1-t)^{2}}=\frac{1}{1-4 t} .
$$

This is exactly the matrix identity (4).

Example 9. The lower triangular matrix in (6) can be written as

$$
\left(C(t), t C(t)^{2}\right)=\left(\frac{1-\sqrt{1-4 t}}{2 t}, \frac{1-2 t-\sqrt{1-4 t}}{2 t}\right) .
$$

The generating functions of its $A$ - and $Z$-sequence are $A(t)=$ $(1+t)^{2}, Z(t)=1+t$. Since $(A(t)-t Z(t)) /(A(t)-4 t)=$ $(1+t) /(1-t)^{2}=\sum_{n=0}^{\infty}(2 n+1) t^{n}$, from Theorem 5, we have

$$
\left(C(t), t C(t)^{2}\right) \frac{1+t}{(1-t)^{2}}=\frac{1}{1-4 t} .
$$

This is exactly the matrix identity (6).

\section{Inverse Relations Determined by Catalan Matrices}

Let $a, b$ be integer numbers, and let $r$ be arbitrary parameter. We define the generalized Catalan matrix $C[a, b ; r]$ to be the Riordan array

$$
C[a, b ; r]=\left(C(r t)^{a}, t C(r t)^{b}\right),
$$

where $C(t)$ is the generating function of Catalan sequence defined in (2). From [27], we have $C(t)^{a}=\sum_{n=0}^{\infty}$ $(a /(2 n+a))\left(\begin{array}{c}2 n+a \\ n\end{array}\right) t^{n}$ for any integer number $a$. Hence $\left[t^{n}\right] C(r t)^{a}\left(t C(r t)^{b}\right)^{k}=\left[t^{n-k}\right] C(r t)^{a+b k}=((a+b k) /(2 n-$ $2 k+a+b k))\left(\begin{array}{c}2 n-2 k+a+b k \\ n-k\end{array}\right) r^{n-k}$. Therefore, by (8), the generic element of the generalized Catalan matrix $C[a, b ; r]$ is given by

$$
C[a, b ; r]_{n, k}=\frac{a+b k}{2 n-2 k+a+b k}\left(\begin{array}{c}
2 n-2 k+a+b k \\
n-k
\end{array}\right) r^{n-k} .
$$

Denote $C[a, b]=C[a, b ; 1]=\left(C(t)^{a}, t C(t)^{b}\right)$. For $0 \leq a, b \leq$ 2 , the corresponding matrices $C[a, b]$ are widely studied by many authors $[5,12,14,15,28,29]$. For example, $C[1,0]=$ $(C(t), t)=((1-\sqrt{1-4 t}) / 2 t, t), C[0,1]=(1, t C(t)), C[2,0]=$ $\left(C(t)^{2}, t\right)$, and $C[0,2]=\left(1, t C(t)^{2}\right)=(1, C(t)-1)$. The matrix $C[1,1]=(C(t), t C(t))$ is the Catalan triangle introduced by Aigner [11] and studied in $[6,12,13]$. The matrix $C[2,2]=$ $\left(C(t)^{2}, t C(t)^{2}\right)$ is the Catalan triangle defined by Shapiro [5]; see also (4). The matrix $C[1,2]=\left(C(t), t C(t)^{2}\right)$ is the Catalan matrix defined by Radoux [9]; see also (6). 
Theorem 10. Let $\left\{F_{n}(x, r)\right\}$ be the polynomial sequence associated to the Riordan array $C[1,1 ; r]^{-1}=(C(r t), t C(r t))^{-1}$. Then, the identities

$$
\begin{gathered}
\sum_{k=0}^{n} \frac{k+1}{n+1}\left(\begin{array}{c}
2 n-k \\
n-k
\end{array}\right) r^{n-k} F_{k}(x, r)=x^{n} \\
\sum_{k=0}^{n}\left(\begin{array}{c}
k+1 \\
n-k
\end{array}\right) x^{k}(-r)^{n-k}=F_{n}(x, r),
\end{gathered}
$$

hold for every $n \in \mathbb{N}$.

Proof. From generic term given in (27) with $a=1$ and $b=1$, we have the generic term of Catalan matrix $C[1,1 ; r]=$ $(C(r t), t C(r t))$ which is $C[1,1 ; r]_{n, k}=((1+k) /(2 n-$ $2 k+1+k))\left(\begin{array}{c}2 n-2 k+1+k \\ n-k\end{array}\right) r^{n-k}$, and by simplifying we obtain $C[1,1 ; r]_{n, k}=((k+1) /(n+1))\left(\begin{array}{c}2 n-k \\ n-k\end{array}\right) r^{n-k}$. Using (11) and (14), we get $C[1,1 ; r]^{-1}=(1-r t, t(1-r t))$, and the generating functions of its $A$ - and $Z$-sequences are $A(t)=1 /(1-r t)$ and $Z(t)=r /(1-r t)$. By Corollary $6, \sum_{n=0}^{\infty} F_{n}(x, r) t^{n}=$ $(A(t)-t Z(t)) /(A(t)-x t)=(1-r t) /\left(1-x t+x r t^{2}\right)$. Therefore, $F_{0}(x, r)=1, F_{1}(x, r)=x-r$, and $F_{n}(x, r)=x F_{n-1}(x, r)-$ $x r F_{n-2}(x, r)$, for $n \geq 2$. Solving this recurrence relation, we have $F_{n}(x, r)=\sum_{k=0}^{n}\left(\begin{array}{c}k+1 \\ n-k\end{array}\right) x^{k}(-r)^{n-k}$. From Corollary 7 , we get the results.

For the case $r=1, x=4$, we have $\sum_{n=0}^{\infty} F_{n}(4,1) t^{n}=(1-$ $t) /\left(1-4 t+4 t^{2}\right)=(1-t) /(1-2 t)^{2}=\sum_{n=0}^{\infty} 2^{n-1}(n+2) t^{n}$. Thus, $F_{n}(4,1)=2^{n-1}(n+2)$. By Theorem 10 , we obtain

$$
\begin{gathered}
\sum_{k=0}^{n}\left(\begin{array}{l}
k+1 \\
n-k
\end{array}\right) 4^{k}(-1)^{n-k}=2^{n-1}(n+2) \\
\sum_{k=0}^{n} \frac{k+1}{n+1}\left(\begin{array}{c}
2 n-k \\
n
\end{array}\right) 2^{k-1}(k+2)=4^{n}
\end{gathered}
$$

The last identity is equivalent to the following matrix identity:

$$
\left(\begin{array}{ccccccc}
1 & 0 & 0 & 0 & 0 & 0 & \cdots \\
1 & 1 & 0 & 0 & 0 & 0 & \cdots \\
2 & 2 & 1 & 0 & 0 & 0 & \cdots \\
5 & 5 & 3 & 1 & 0 & 0 & \cdots \\
14 & 14 & 9 & 4 & 1 & 0 & \cdots \\
42 & 42 & 28 & 14 & 5 & 1 & \cdots \\
\vdots & \vdots & \vdots & \vdots & \vdots & \vdots & \ddots
\end{array}\right)\left(\begin{array}{c}
1 \\
3 \\
8 \\
20 \\
48 \\
112 \\
\vdots
\end{array}\right)=\left(\begin{array}{c}
1 \\
4 \\
4^{2} \\
4^{3} \\
4^{4} \\
4^{5} \\
\vdots
\end{array}\right)
$$

Theorem 11. Let $\left\{u_{n}(x, r)\right\}$ be the polynomial sequence associated to the Riordan array $C[1,2 ; r]^{-1}=\left(C(r t), t C(r t)^{2}\right)^{-1}$. Then, for any nonnegative integer $n$, one has

$$
\begin{gathered}
\sum_{k=0}^{n} \frac{2 k+1}{2 n+1}\left(\begin{array}{c}
2 n+1 \\
n-k
\end{array}\right) r^{n-k} u_{k}(x, r)=x^{n} \\
\sum_{k=0}^{n}\left(\begin{array}{c}
n+k \\
n-k
\end{array}\right) x^{k}(-r)^{n-k}=u_{n}(x, r) .
\end{gathered}
$$

Proof. From generic term given in (27) with $a=1$ and $b=2$, we have the generic term of Catalan matrix $C[1,2 ; r]$ $=\left(C(r t), t C(r t)^{2}\right)$ which is $C[1,2 ; r]_{n, k}=((2 k+1) /(2 n+$ 1)) $\left(\begin{array}{c}2 n+1 \\ n-k\end{array}\right) r^{n-k}$. Using (11), we obtain $C[1,2 ; r]^{-1}=(1 /(1+$ $\left.r t), t /(1+r t)^{2}\right)$. Hence, the generic term of $C[1,2 ; r]^{-1}$ is $\left[t^{n}\right](1 /(1+r t))\left(t /(1+r t)^{2}\right)^{k}=\left[t^{n-k}\right](1 /(1+r t))^{2 k+1}=$ $(-r)^{n-k}\left(\begin{array}{c}n+k \\ n-k\end{array}\right)$. By Corollary 7 , we obtain the desired results.

Since $C[1,2 ; r]^{-1}=\left(1 /(1+r t), t /(1+r t)^{2}\right)$, from Lemma 2 , the generating functions of $A$ - and $Z$-sequences of $C[1,2 ; r]$ are $A(t)=(1+r t)^{2}$ and $Z(t)=r+r^{2} t$. Hence, $\sum_{k=0}^{n} u_{n}(x, r) t^{n}=$ $(A(t)-t Z(t)) /(A(t)-x t)=(1+r t) /\left(1-(x-2 r) t+r^{2} t^{2}\right)$. For the case $r=1$ and $x=4$, we have $\sum_{k=0}^{n} u_{n}(4,1) t^{n}=(1+t) /(1-$ $\left.2 t+t^{2}\right)=(1+t) /(1-t)^{2}=\sum_{k=0}^{n}(2 n+1) t^{n}$, and $u_{n}(4,1)=2 n+1$. By Theorem 11, we have

$$
\begin{gathered}
\sum_{k=0}^{n}(-1)^{n-k}\left(\begin{array}{c}
n+k \\
n-k
\end{array}\right) 4^{k}=2 n+1, \\
\sum_{k=0}^{n} \frac{2 k+1}{2 n+1}\left(\begin{array}{c}
2 n+1 \\
n-k
\end{array}\right)(2 k+1)=4^{n} .
\end{gathered}
$$

The last identity is equivalent to identity (6).

For the case $r=-1$ and $x=1$, we have $\sum_{k=0}^{n} u_{n}(1,-1) t^{n}=$ $(1-t) /\left(1-3 t+t^{2}\right)=\sum_{k=0}^{n} F_{2 n+1} t^{n}$, and $u_{n}(1,-1)=F_{2 n+1}$, where $\left\{F_{n}\right\}_{n \geq 0}$ is Fibonacci sequence with generating $\sum_{k=0}^{\infty} F_{n} t^{n}=$ $t /\left(1-t-t^{2}\right)$. By Theorem 11, we have

$$
\begin{gathered}
\sum_{k=0}^{n}(-1)^{n-k} \frac{2 k+1}{2 n+1}\left(\begin{array}{c}
2 n+1 \\
n-k
\end{array}\right) F_{2 k+1}=1 \\
\sum_{k=0}^{n}\left(\begin{array}{c}
n+k \\
n-k
\end{array}\right) 4^{k}=F_{2 n+1}
\end{gathered}
$$

Theorem 12. Let $\left\{v_{n}(x, r)\right\}$ be the polynomial sequence associated to the Riordan array $C[2,2 ; r]^{-1}=\left(C(r t)^{2}, t C(r t)^{2}\right)^{-1}$. Then, one has

$$
\begin{aligned}
& \sum_{k=0}^{n} \frac{k+1}{n+1}\left(\begin{array}{c}
2 n+2 \\
n-k
\end{array}\right) r^{n-k} v_{k}(x, r)=x^{n}, \\
& \sum_{k=0}^{n}\left(\begin{array}{c}
n+k+1 \\
n-k
\end{array}\right) x^{k}(-r)^{n-k}=v_{n}(x, r),
\end{aligned}
$$

for every $n \in \mathbb{N}$.

Proof. From generic term given in (27) with $a=2$ and $b=2$, we have the generic term of Catalan matrix $C[2,2 ; r]=\left(C(r t)^{2}, t C(r t)^{2}\right)$ which is $C[2,2 ; r]_{n, k}=((k+1) /$ $(n+1))\left(\begin{array}{c}2 n+2 \\ n-k\end{array}\right) r^{n-k}$. Using (11), we obtain $C[2,2 ; r]^{-1}$ $=\left(1 /(1+r t)^{2}, t /(1+r t)^{2}\right)$. Hence, the generic term of $C[2,2 ; r]^{-1}$ is $\left[t^{n}\right]\left(1 /(1+r t)^{2}\right)\left(t /(1+r t)^{2}\right)^{k}=$ $\left[t^{n-k}\right](1 /(1+r t))^{2 k+2}=(-r)^{n-k}\left(\begin{array}{c}n+k+1 \\ n-k\end{array}\right)$. By Corollary 7 , we obtain the desired results. 
The generating functions of $A$ - and $Z$-sequences of $C[2,2 ; r]$ are $A(t)=1+2 r t+r^{2} t^{2}$ and $Z(t)=2 r+r^{2} t$. Thus $\sum_{n=0}^{\infty} v_{n}(x, r) t^{n}=(A(t)-t Z(t)) /(A(t)-x t)=1 /(1-(x-2 r) t+$ $\left.r^{2} t^{2}\right)$.

For the case $r=1$ and $x=2 y+2$, we have $\sum_{n=0}^{\infty} v_{n}(2 y+$ $2,1) t^{n}=1 /\left(1-2 y t+t^{2}\right)=\sum_{n=0}^{\infty} U_{n}(y) t^{n}$, where $U_{n}(y)$ are Chebyshev polynomials of the second kind (see [1]). Hence, by Theorem 13, we have

$$
\begin{gathered}
\sum_{k=0}^{n}\left(\begin{array}{c}
n+k+1 \\
n-k
\end{array}\right)(2 y+2)^{k}(-1)^{n-k}=U_{n}(y), \\
\sum_{k=0}^{n} \frac{k+1}{n+1}\left(\begin{array}{c}
2 n+2 \\
n-k
\end{array}\right) U_{k}(y)=(2 y+2)^{n} .
\end{gathered}
$$

Substituting $y=1$ in the last identity, we get (4) again.

Theorem 13. Let $\left\{w_{n}(x, r)\right\}$ be the polynomial sequence associated to the Riordan array $C[2,1 ; r]^{-1}=\left(C(r t)^{2}, t C(r t)\right)^{-1}$. Then, one has identities

$$
\begin{gathered}
\sum_{k=0}^{n} \frac{k+2}{n+2}\left(\begin{array}{c}
2 n-k+1 \\
n-k
\end{array}\right) r^{n-k} w_{k}(x, r)=x^{n} \\
\sum_{k=0}^{n}\left(\begin{array}{c}
k+2 \\
n-k
\end{array}\right) x^{k}(-r)^{n-k}=w_{n}(x, r) .
\end{gathered}
$$

Proof. Using (11), we obtain $C[2,1 ; r]^{-1}=\left(C(r t)^{2}, t C(r t)\right)^{-1}$ $=\left((1-r t)^{2}, t-r t^{2}\right)$. Hence, the generic term of $C[2,2 ; r]^{-1}$ is $\left[t^{n}\right](1-r t)^{2}\left(t-r t^{2}\right)^{k}=\left[t^{n-k}\right](1-r t)^{k+2}=(-r)^{n-k}\left(\begin{array}{c}k+2 \\ n-k\end{array}\right)$. From generic term given in (27) with $a=2$ and $b=1$, we have the generic term of Catalan matrix $C[2,1 ; r]=$ $\left(C(r t)^{2}, t C(r t)\right)$ which is $C[2,1 ; r]_{n, k}=((2+k) /(2 n-$ $2 k+2+k))\left(\begin{array}{c}2 n-2 k+2+k \\ n-k\end{array}\right) r^{n-k}$, and by simplifying we obtain $C[1,1 ; r]_{n, k}=((k+2) /(n+2))\left(\begin{array}{c}2 n-k+1 \\ n-k\end{array}\right) r^{n-k}$. From Corollary 7 , we obtain the desired results.

The generating functions for the $A$ - and $Z$-sequences of $C[2,1 ; r]$ are $A(t)=1 /(1-r t), Z(t)=\left(2 r-r^{2} t\right) /(1-r t)$, and $\sum_{n=0}^{\infty} w_{n}(x, r) t^{n}=(A(t)-t Z(t)) /(A(t)-x t)=(1-r t)^{2} /(1-x t+$ $\left.x r t^{2}\right)$. For the case $r=1$ and $x=4$, we have $\sum_{n=0}^{\infty} w_{n}(4,1) t^{n}=$ $(1-t)^{2} /\left(1-4 t+4 t^{2}\right)=(1-t)^{2} /(1-2 t)^{2}=1+\sum_{n=1}^{\infty}(n+3) 2^{n-2} t^{n}$. By Theorem 13, we have

$$
\begin{gathered}
\sum_{k=0}^{n}(-1)^{n-k}\left(\begin{array}{l}
k+2 \\
n-k
\end{array}\right) 4^{k}=(n+3) 2^{n-2}, \quad n \geq 1, \\
\frac{2}{n+2}\left(\begin{array}{c}
2 n+1 \\
n+1
\end{array}\right)+\sum_{k=1}^{n} \frac{k+2}{n+2}\left(\begin{array}{c}
2 n-k+1 \\
n+1
\end{array}\right)(k+3) 2^{k-2} \\
=4^{n}, \quad n \geq 0 .
\end{gathered}
$$

The matrix form of the last identity is

$$
\left(\begin{array}{cccccc}
1 & 0 & 0 & 0 & 0 & \cdots \\
2 & 1 & 0 & 0 & 0 & \cdots \\
5 & 3 & 1 & 0 & 0 & \cdots \\
14 & 9 & 4 & 1 & 0 & \cdots \\
42 & 28 & 14 & 5 & 1 & \cdots \\
\vdots & \vdots & \vdots & \vdots & \vdots & \ddots
\end{array}\right)\left(\begin{array}{c}
1 \\
2 \\
5 \\
12 \\
28 \\
\vdots
\end{array}\right)=\left(\begin{array}{c}
1 \\
4 \\
4^{2} \\
4^{3} \\
4^{4} \\
\vdots
\end{array}\right) .
$$

\section{Acknowledgments}

The author would like to thank the referees for their helpful suggestions. This work was supported by the National Natural Science Foundation of China (11261032).

\section{References}

[1] L. Comtet, Advanced Combinatorics, D. Reidel Publishing, Dordrecht, The Netherlands, 1974.

[2] R. P. Stanley, Enumerative Combinatorics, vol. 2, Cambridge University Press, Cambridge, UK, 1999.

[3] D. G. Rogers, "Pascal triangles, Catalan numbers and renewal arrays," Discrete Mathematics, vol. 22, no. 3, pp. 301-310, 1978.

[4] C. L. Mallows and L. Shapiro, "Balls on the lawn," Journal of Integer Sequences, vol. 2, article 99.1.5, 1999.

[5] L. W. Shapiro, “A Catalan triangle," Discrete Mathematics, vol. 14, no. 1, pp. 83-90, 1976.

[6] L. W. Shapiro, W.-J. Woan, and S. Getu, "Runs, slides and moments," SIAM Journal on Algebraic Discrete Methods, vol. 4, no. 4, pp. 459-466, 1983.

[7] W.-J. Woan, L. Shapiro, and D. G. Rogers, "The Catalan numbers, the Lebesgue integral, and $4^{n-2}$," The American Mathematical Monthly, vol. 104, no. 10, pp. 926-931, 1997.

[8] Y. C. Chen, Y. Li, L. W. Shapiro, and H. F. Yan, "Matrix identities on weighted partial Motzkin paths," European Journal of Combinatorics, vol. 28, no. 4, pp. 1196-1207, 2007.

[9] C. Radoux, "Additional formulas for polynomials built on classical combinatorial sequences," Journal of Computational and Applied Mathematics, vol. 115, no. 1-2, pp. 471-477, 2000.

[10] E. Deng and W. J. Yan, "Some identities on the Catalan, Motzkin and Schröder numbers," Discrete Applied Mathematics, vol. 156, no. 14, pp. 2781-2789, 2008.

[11] M. Aigner, "Enumeration via ballot numbers," Discrete Mathematics, vol. 308, no. 12, pp. 2544-2563, 2008.

[12] T. X. He, "Parametric Catalan numbers and Catalan triangles," Linear Algebra and Its Applications, vol. 438, no. 3, pp. 14671484, 2013.

[13] G. S. Cheon, H. Kim, and L. W. Shapiro, "Combinatorics of Riordan arrays with identical $A$ and $Z$ sequences," Discrete Mathematics, vol. 312, no. 12-13, pp. 2040-2049, 2012.

[14] S. Stanimirović, P. Stanimirović, and A. Ilić, "Ballot matrix as Catalan matrix power and related identities," Discrete Applied Mathematics, vol. 160, no. 3, pp. 344-351, 2012.

[15] L. W. Shapiro, S. Getu, W.-J. Woan, and L. Woodson, "The Riordan group," Discrete Applied Mathematics, vol. 34, no. 1-3, pp. 229-239, 1991.

[16] R. Sprugnoli, "Riordan arrays and combinatorial sums," Discrete Mathematics, vol. 132, no. 1-3, pp. 267-290, 1994. 
[17] P. Peart and L. Woodson, "Triple factorization of some Riordan matrices," The Fibonacci Quarterly, vol. 31, no. 2, pp. 121-128, 1993.

[18] P. Peart and W. Woan, "A divisibility property for a subgroup of Riordan matrices," Discrete Applied Mathematics, vol. 98, no. 3, pp. 255-263, 2000.

[19] P. Barry, "A note on a one-parameter family of Catalan-like numbers," Journal of Integer Sequences, vol. 12, no. 5, article 09.5.4, 2009.

[20] N. T. Cameron and A. Nkwanta, "On some (pseudo) involutions in the Riordan group," Journal of Integer Sequences, vol. 8, no. 3, article 05.3.7, 2005.

[21] A. Luzón, D. Merlini, M. Morón, and R. Sprugnoli, "Identities induced by Riordan arrays," Linear Algebra and Its Applications, vol. 436, no. 3, pp. 631-647, 2012.

[22] T. X. He and R. Sprugnoli, "Sequence characterization of Riordan arrays," Discrete Mathematics, vol. 309, no. 12, pp. 39623974, 2009.

[23] D. Merlini, D. G. Rogers, R. Sprugnoli, and M. C. Verri, "On some alternative characterizations of Riordan arrays," Canadian Journal of Mathematics, vol. 49, no. 2, pp. 301-320, 1997.

[24] M. Bayat and H. Teimoori, "Pascal $k$-eliminated functional matrix and its property," Linear Algebra and Its Applications, vol. 308, no. 1-3, pp. 65-75, 2000.

[25] S.-L Yang and S.-N. Zheng, "Determinant representations of polynomial sequences of Riordan type," Journal of Discrete Mathematics, vol. 2013, Article ID 734836, 6 pages, 2013.

[26] A. Luzón and M. A. Morón, "Recurrence relations for polynomial sequences via Riordan matrices," Linear Algebra and Its Applications, vol. 433, no. 7, pp. 1422-1446, 2010.

[27] R. Graham, D. Knuth, and O. Patashnik, Concrete Mathematics, Addison-Wesley, New York, NY, USA, 1989.

[28] A. Nkwanta and E. Barnes, "Two Catalan-type Riordan arrays and their connections to the Chebyshev polynomials of the first kind," Journal of Integer Sequences, vol. 15, no. 3, article 12.3.3, 2012.

[29] S. Stanimirović, P. Stanimirović, M. Miladinović, and A. Ilić, "Catalan matrix and related combinatorial identities," Applied Mathematics and Computation, vol. 215, no. 2, pp. 796-805, 2009. 


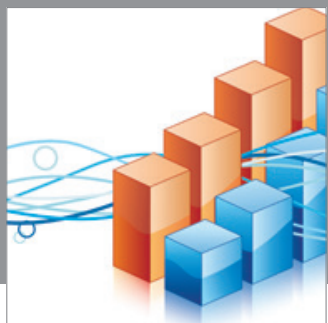

Advances in

Operations Research

mansans

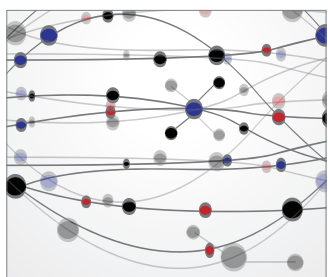

The Scientific World Journal
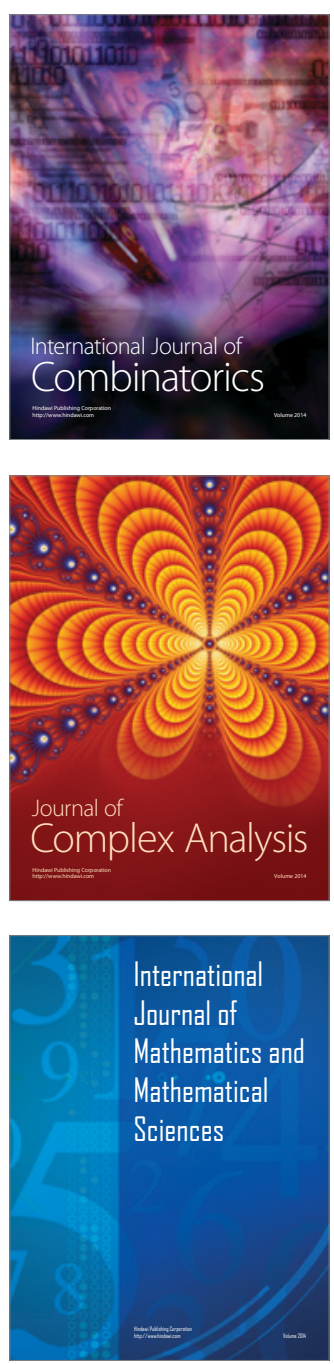
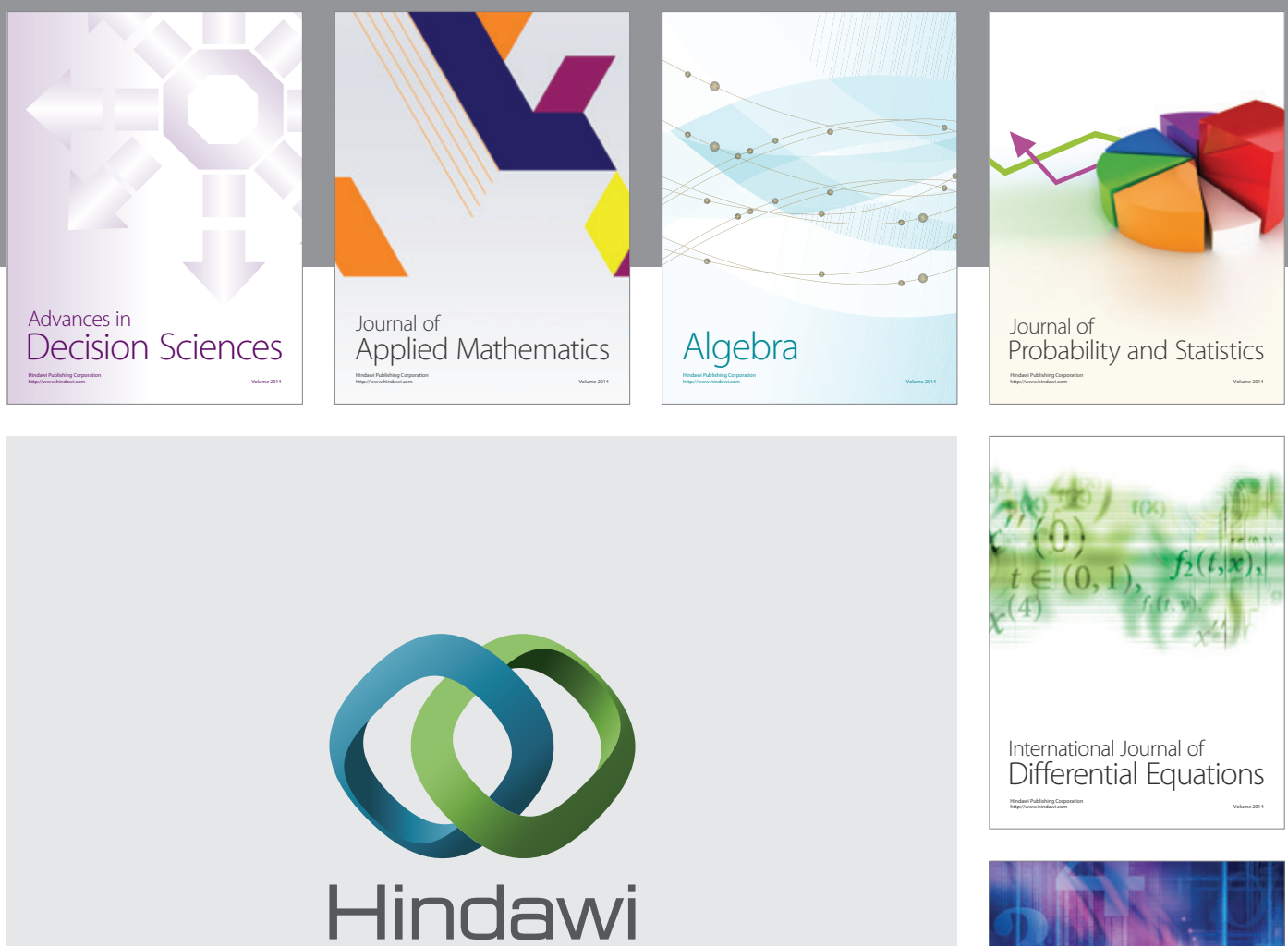

Submit your manuscripts at http://www.hindawi.com
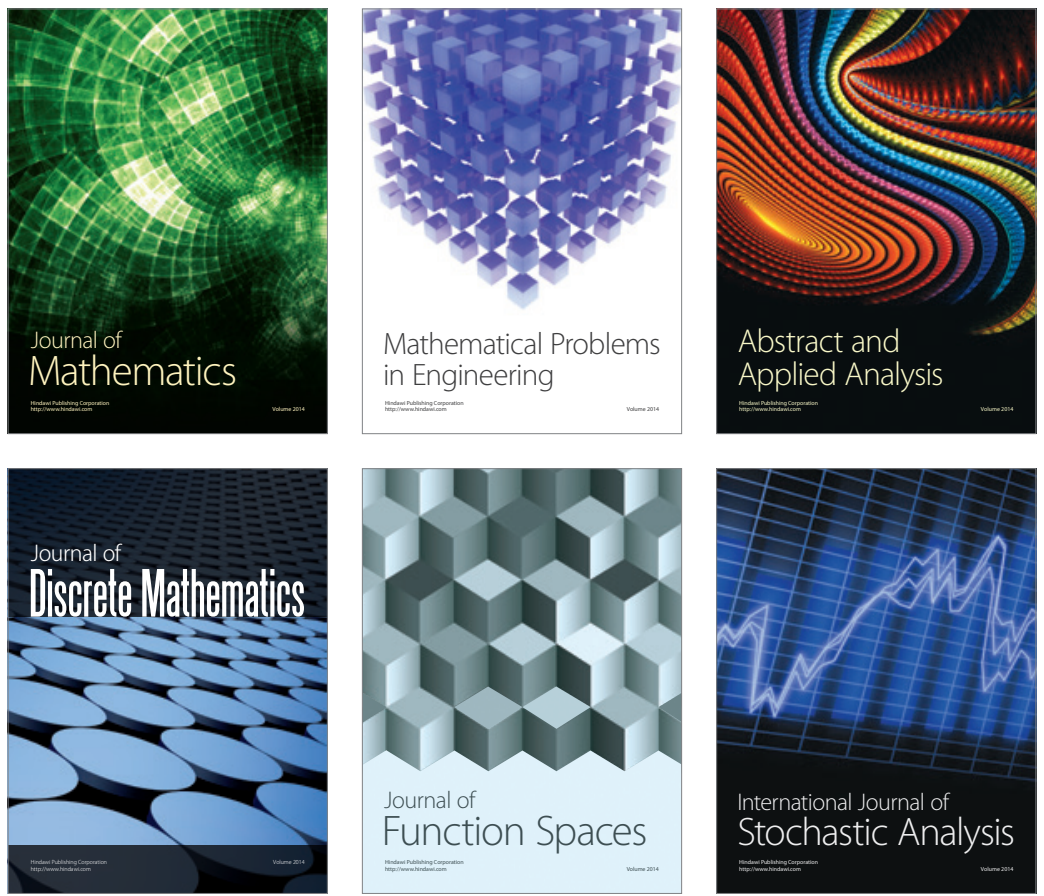

Journal of

Function Spaces

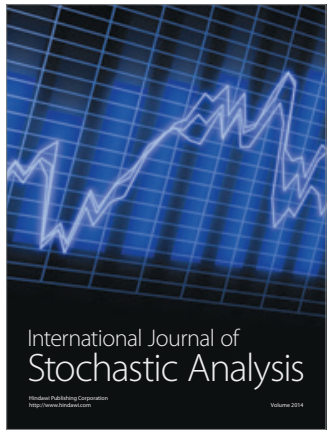

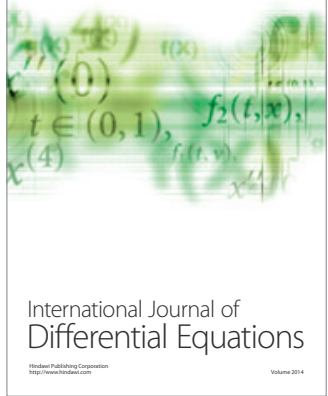
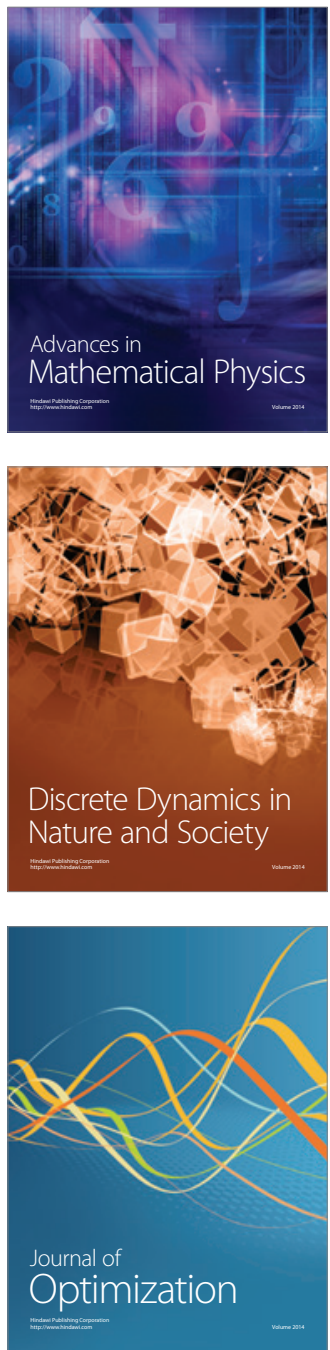